\title{
Influence of boron on the growth and biochemical changes in plant growth promoting rhizobacteria (PGPR) inoculated banana plantlets
}

\begin{abstract}
The effects of rhizobacteria inoculation in modified MS medium containing boron ( 1 and 10 $\mathrm{lM}$ ) on the biochemical components, physiological characteristics and mineral content of the in vitro banana plantlets were carried out. The presence of rhizobacteria in the medium supplemented with boron at two concentrations: 1 and $10 \mathrm{lM}$ resulted in an improvement in growth and root biomass compared to the control (uninoculated). Rhizobacteria inoculation also produced an increase in protein, nitrate, soluble nitrogen and chlorophyll contents of the plantlets cultured in MS modified medium containing boron. An increase in percentage of growth ([295\%) was shown when boron was applied into medium inoculated with Bacillus sphaericus UPMB10. The effectiveness of inoculation is increased when associated with boron, nitrogen or carbon into the medium. Thus, these bacterial strains could be used as a bioenhancer for growth of in vitro banana plantlets.
\end{abstract}

Keyword: Rhizobacteria, Banana, Boron, Growth rate, Biochemical analysis 